\title{
SOME THEOREMS ON THE AUTOMORPHISM GROUPS OF PLANAR TERNARY RINGS
}

\section{REUBEN SANDLER}

In [4], Knuth develops the notion of isotopy for general ternary rings which coordinatize projective planes, and proves several theorems analogous to the theorems proved by Albert [1] for nonassociative division rings. He then defines the concept of an autotopism of a ternary ring, and demonstrates the isomorphism between the autotopism group of a ternary ring $\Re$ and a certain subgroup of the collineation group of the projective plane coordinatized by $\Re$. In this note, a slight extension of Knuth's ideas is used to obtain some information about the automorphism groups of ternary rings. For completeness Knuth's result on autotopism groups (Theorem 1) will be proved independently here so as to make the proof of Theorem 2 self-contained.

The notation and terminology used is the same as in Hall [3, Chapter 20]. In particular, the reader is assumed to be familiar with Hall's discussion of coordinatization, ternary rings, and central collineations.

Let $\pi$ be a projective plane, and let $(\infty),(0),(0,0)$, and $(1,1)$ be any four points of $\pi$, no three of which are collinear. Then, as in [3], we can coordinatize $\pi$ and obtain a ternary ring $\Re$, in general depending on the four points selected. If $\gamma$ is a collineation of $\pi$ such that

$$
(\infty) \gamma=(\infty), \quad(0) \gamma=(0), \quad(0,0) \gamma=(0,0),
$$

then we must have

$\left(1^{\prime}\right) \quad\left[L_{\infty}\right] \gamma=\left[L_{\infty}\right], \quad[x=0] \gamma=[x=0], \quad[y=0] \gamma=[y=0]$.

Thus, $\gamma$ defines three one-one permutations, $Q, P, U$, of the elements of $\Re$, by

$$
\begin{aligned}
(x, 0) \gamma & =(x Q, 0), & & (0, y) \gamma=(0, y U), \\
(m) \gamma & =(m P), & & (x, y) \gamma=(x Q, y U),
\end{aligned}
$$

where, by (1), we must have $0 Q=0 P=0 U=0$. Now,

$$
(x, y) \in[m, k] \Leftrightarrow y=x \cdot m \circ k
$$

Received by the editors July 12, 1963. 
(where $[m, k]$ is the line determined by $(m)$ and $(0, k)$ ), and since $\gamma$ is a collineation, we have

$$
\begin{aligned}
(x, y) \gamma \in[m, k] \gamma & \Leftrightarrow(x Q, y U) \in[m P, k U] \\
& \Leftrightarrow y U=x Q \cdot m P \circ k U=(x \cdot m \circ k) U .
\end{aligned}
$$

A triple of one-one transformations $(Q, P, U)$ of $\Re$ onto $\Re$ is called an autotopism of $\Re$ if

$$
\begin{gathered}
0 Q=0 P=0 U=0, \\
(x \cdot m \circ k) U=x Q \cdot m P \circ k U .
\end{gathered}
$$

Any autotopism of $\Re$ can be used to define a collineation of $\pi$, $\gamma(Q, P, U)$, by means of (2). Notice now that

$$
\gamma(Q, P, U) \cdot \gamma\left(Q_{1}, P_{1}, U_{1}\right)=\gamma\left(Q Q_{1}, P P_{1}, U U_{1}\right),
$$

and we have demonstrated the truth of

Theorem 1 (KNUTH). Let $\pi$ be a projective plane, and let $\Re$ be one of the coordinatizing ternary rings for $\pi$ determined by $(\infty),(0)$, and $(0,0)$ ( $\Re$ will be uniquely determined by the further choice of the point $(1,1))$. Then the autotopism group of $\Re$ is isomorphic with the subgroup of collineations of $\pi$ which fix the three points $(\infty),(0)$, and $(0,0)$.

Now, in (4), let $k=0$, and obtain $(x m) U=(x Q)(m P)$. Thus, letting first $m=1$, then $x=1$, we have

$$
U=Q R_{1 P}=P L_{1 Q},
$$

where the operators $R$ and $L$ are defined by $x y=x R_{y}=y L_{x}$. Thus, if $Q=P=U$, we have, from (5),

$$
1 Q=1 P=1 U=1 \text {. }
$$

Also, when $Q=P=U$, if $m=1$ in (4), we have $(x+k) U=x U+k U$, while if $k=0$ in (4), we have $(x m) U=(x U)(m U)$. So, if we define an automorphism of a ternary ring in the natural way, as an autotopism in which $Q=P=U$, we have a definition which is as desired on the two binary operations which a ternary ring defines. From Theorem 1, then, and equations (2), (5) and (6), we have immediately

TheOREM 2. Let $\pi$ be a projective plane, and let $\Re$ be the ternary ring coordinatizing $\pi$ and defined by the points $(\infty),(0),(0,0)$, and $(1,1)$. Then the automorphism group of $\Re$ is isomorphic with the subgroup of collineations of $\pi$ which leave all four points fixed.

Given any four points in a projective plane, $A, B, C, D$, no three 
of them collinear, there are in general 24 distinct (nonisomorphic) coordinatizing ternary rings defined by these four points, depending on which of the four is designated as $(\infty)$, which as $(0)$, which as $(0,0)$, and which as $(1,1)$. Except for certain very special cases, nothing is known about the algebraic relationships between these 24 rings. The following immediate corollary to Theorem 2 gives some information concerning this question.

Theorem 3. Let $\pi$ be a projective plane, and let $A, B, C, D$ be four points of $\pi$, no three of which are collinear. Then the 24 coordinatizing ternary rings of $\pi$ determined by $A, B, C, D$ have isomorphic automorphism groups.

It is, of course, not true in general that all coordinatizing rings for a plane have isomorphic automorphism or autotopism groups. Also, in some cases, the various rings defined by four points can look completely different and have no recognizable relationships-some being, for example, V-W systems, while some are nonlinear.

For the proof of the next theorem, which concerns finite projective planes, the following result will be necessary. Recall that in a finite projective plane $\pi$, every line contains $n+1$ points, where $n$ is called the order of $\pi$.

LEMMA 1 (BAER [2]). Let $\pi$ be a finite projective plane of order $n$. Then if $\alpha$ is an involutory collineation of $\pi\left(\alpha^{2}=I\right)$, then either $\alpha$ is a central collineation (i.e., $\alpha$ fixes all of the points on some line $L$, and at most one point not on $L$ ) or the set of points and lines fixed by $\alpha$ forms $a$ subplane of $\pi, \pi_{0}$, which is of order $\sqrt{ } n$.

As a corollary of Lemma 1 and Theorem 2, we have

THEOREM 4. Let $\pi$ be a finite projective plane of order $n$, where $n \neq m^{2}$ for any integer $m$. Then if $\Re$ is any coordinatizing ring of $\pi$, the automorphism group of $\mathfrak{R}$ has odd order.

Proof. Let $\phi$ be an automorphism of $\Re$ such that $\phi^{2}=I$. Then, by Theorem 2, there is an involutory collineation, $\alpha$, of $\pi$ fixing the four points $(\infty),(0),(0,0),(1,1)$ defining $\Re$. Since no three of these points are collinear, $\alpha$ cannot be a central collineation. Thus, by Lemma $1, \alpha$ must fix a subplane of $\pi$ which has order $\sqrt{ } n$. By hypothesis, however, this is impossible, so $\phi^{2}=I$ implies $\phi=I$, and the automorphism group must have odd order.

In the case where $\Re$ is a nonassociative division ring, Theorem 4 can be proved directly by algebraic methods. An interesting feature of the theorem, however, is that it seems to be the case that this 
algebraic theorem can be proved in its full generality only by resorting to the geometric lemma. This is in direct contrast to the situation where one can prove the geometric theorem that a finite Desarguesian plane satisfies Pappus's theorem only by using the algebraic fact that a finite division ring is commutative.

\section{REFERENCES}

1. A. A. Albert, Finite division algebras and finite planes, Combinatorial analysis, Proc. Sympos. Appl. Math. Vol. 10, pp. 53-70, Amer. Math. Soc., Providence, R. I., 1960.

2. R. Baer, Projectivities with fixed points on every line of the plane, Bull. Amer. Math. Soc. 52 (1946), 273-286.

3. Marshall Hall, Jr., The theory of groups, Macmillan, New York, 1959.

4. D. Knuth, Finite semifields and projective planes, Ph.D. Dissertation, California Institute of Technology, Pasadena, Calif., 1963.

InSTitute for Defense Analyses

\section{PARTITIONS WITH EQUAL PRODUCTS}

JOHN B. KELLY

T. S. Motzkin has conjectured (oral communication) that every sufficiently large positive integer can be partitioned into three positive integral parts in two different ways so that the products of the integers in the two partitions are equal (e.g., $13=1+6+6=2+2+9$; $1 \cdot 6 \cdot 6=2 \cdot 2 \cdot 9=36)$. In this note we prove a generalization of this conjecture.

THEOREM. Let $k$ be an integer $\geqq 3$. There exists an integer $N(k)$ such that every integer $n \geqq N(k)$ can be partitioned into $k$ parts in $k-1$ different ways:

$$
\begin{aligned}
n & =a_{11}+\cdots+a_{1 k} \\
& =a_{21}+\cdots+a_{2 k}=\cdots=a_{k-1,1}+\cdots+a_{k-1, k},
\end{aligned}
$$

where

(2) $\quad a_{11} \cdot a_{12} \cdot \cdots a_{1 k}=a_{21} \cdot a_{22} \cdot \cdots a_{2 k}=\cdots=a_{k-1,1} \cdot \cdots \cdot a_{k-1 . k}$

Received by the editors July 26, 1963. 